\title{
Choroid Melanoma Metastasis to Lumbar Spine: A Case Report
}

\author{
Yeo Song Kim, Jeong Gyun Kim, Jong Joo Rhee, Jong Won Lee, Hyun Koo Lee, Jin Woo Hur \\ Department of Neurosurgery, Cheongju St. Mary's Hospitial, The Catholic University of Korea College of Medicine, \\ Cheongju, Republic of Korea
}

Corresponding author: Jin Woo Hur Department of Neurosurgery, Cheongju St. Mary's Hospitial, The Catholic University of Korea College of Medicine, 173-19, Juseong-ro, Cheongwon-gu, Cheongju 28323, Republic of Korea

Tel: $+82-43-219-8084$

Fax: $+82-43-219-8084$

E-mail: somalh@naver.com

Received: August 16, 2020

Revised: September 10, 2020

Accepted: September 22, 2020
A 57-year-old woman visited our hospital with left flank pain and left anterior thigh and calf radiation pain. A lumbar spine magnetic resonance imaging (MRI) was showed that a heterogenous enhancing ventral epidural mass compressed the dura posteriorly at L3 level and totally enhanced L3 vertebral body. To reduce pain, L3 laminectomy, left facetectomy, and partial tumor removal with posterior pedicle screw fixation were performed. On examination, the tumor was soft and black-colored. After the operation, histopathological assessment confirmed malignant melanoma. The patient had a history of choroid malignant melanoma of the right eye diagnosed 8 years previously. The patient underwent treatment at another hospital and achieved complete remission after 5 years. As follow-up was not performed, the metastasis was not detected earlier. To our knowledge, this is the first report of the lumbar spine as the main lesion location without thoracic or cervical spine symptoms.

Key Words: Choroid; Lumbar vertebrae; Melanoma; Neoplasm metastasis

\section{INTRODUCTION}

Intraocular melanomas are the most common primary ocular malignancy in adults, but accounts for $5 \%$ of all malignant melanomas ${ }^{6}$. Approximately 95\% of melanoma around the eye is found in the uvea, and choroid melanoma is the most common primary ocular malignancy in adults. In many cases, the predominant site of metastatic disease for patients with choroid melanoma is the liver. Spinal/vertebral metastasis is an infrequent clinical entity. Spinal metastasis of choroid malignant melanoma has been reported only in 3 previous cases ${ }^{2,3)}$. However, previous reports have discussed thoracic spine metastasis. To our knowledge, this is the first report of the lumbar spine as the main lesion location without thoracic or cervical spine symptoms.

\section{CASE REPORT}

A 57-year-old woman visited our hospital with left flank pain and left anterior thigh and calf radiation pain that had persisted for one month. There was no history of trauma or fall. She was admitted to the hospital and underwent lumbar spine magnetic resonance imaging (MRI). The T1-weighted image (WI) showed a body fracture line and high-intensity signal at the L3 vertebral body. A low-intensity signal was de- tected in this area on T2-WI, and contrast MRI showed a heterogeneous enhancing ventral epidural mass compressing the dura posteriorly at the L3 level and totally enhanced the L3 vertebral body (Fig. 1A). Positron emission tomography-computed tomography (PET-CT) showed a contrast of the liver, both ribs, clavicle, sternum, thorax-lumbar-sacrum spine, both pelvic bones, right humeral shaft, and left proximal femur (Fig. 1B). To reduce the patient's pain, L3 laminectomy, left facetectomy,
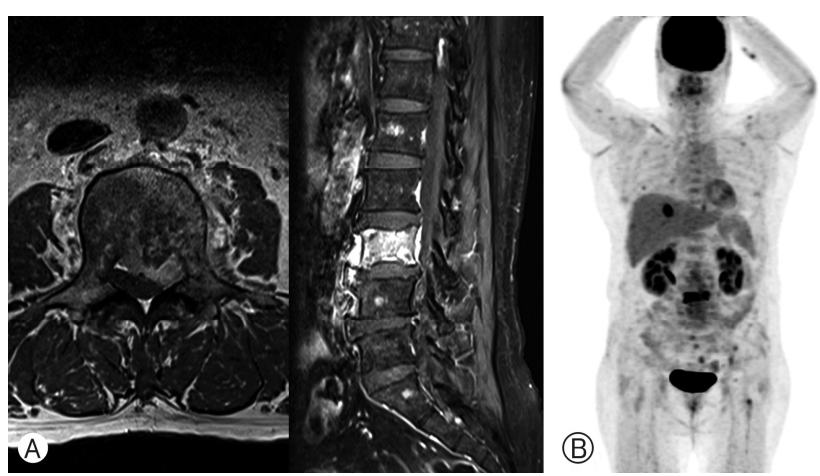

Fig. 1. Preoperative gadolinium-enhanced magnetic resonance imaging. On images, strongly enhanced lumbar 3 vertebral body and posterior vertebral body soft tissue tumor is observed (A). Positron emission tomography-computed tomography showed liver, both ribs, clavicles, sternum, thorax-lumbar-sacrum spines, both pelvic bones, right humeral shaft, left proximal femur was contrasted (B). 
and partial tumor removal with posterior pedicle screw fixation were performed. After L3 laminectomy, the soft and blackcolored mass was presented on vertebral body (Fig. 2A). Immunohistochemistry from the vertebral body showed strongly positive human melanoma black 45 (Fig. 3). And additional histopathological assessment confirmed that it was malignant melanoma (Fig. 2B).
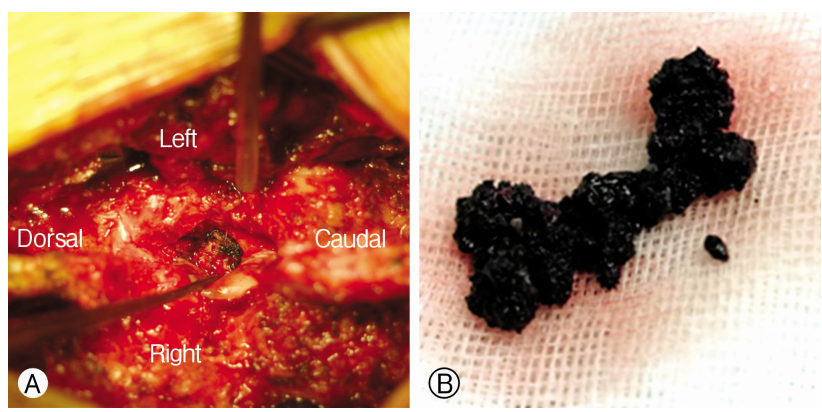

Fig. 2. During operation, after retracting the root with the root retractor, a black mass is visible (A). Curretaged tumor pieces (B).
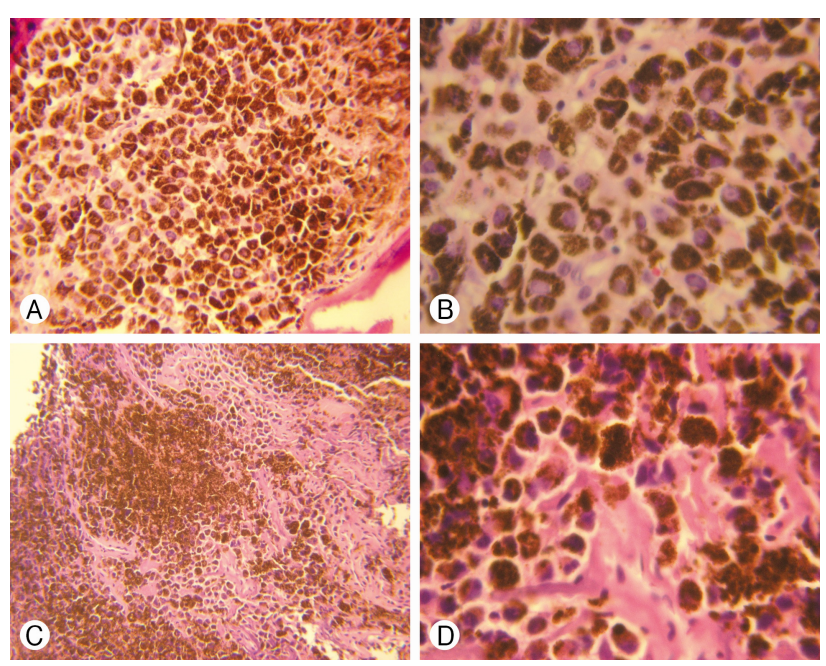

Fig. 3. Histological showing strands of focally pigmented pleomorphic epithelioid cells in decalcified sections of bone features are fully consistent with metastatic melanoma. (A) Hematoxylin and eosin stain (H\&E), $\times 200$ magnification. (B) $H \& E, \times 400$ magnification. (C) Human melanoma black-45 stain (HMB-45), ×100 magnification. (D) HMB-45, ×400 magnification.
The patient had a history of enucleation of the right eyeball that was diagnosed 8 years prior, the pathology of which identified it as choroid malignant melanoma. Family history revealed that her mother died of pancreatic cancer 40 years previous, and her brother died of liver cancer 2 years prior. Several imaging of the choroid malignant melanoma and PETCT were performed in the hospital where she had received the first diagnosis, and she received treatment and follow-up for 5 years. After 5 years, the patient achieved complete remission and refused additional follow-up. Thus metastasis could not detected early.

After the operation, her left leg and flank pain was improved, and she was discharged after 10 days without complication. After surgery, radiotherapy (RTx) and chemotherapy (CTx) are being performed at other hospitals, and they have survived more than 1 year after surgery.

\section{DISCUSSION}

Choroid melanomas are the most common primary intraocular malignant tumor in adults. While choroid melanomas represent only $5 \%$ of all melanomas, they have a high rate of metastasis and poor response to treatment and account for about $13 \%$ of melanoma deaths ${ }^{3)}$. Because there is currently a limited amount of data on spinal metastasis from choroid melanomas, treatment for this is similar to that for all types of melanoma to spinal/vertebral metastasis ${ }^{3)}$.

Several studies have suggested that all therapies for uveal melanoma metastasis do not significantly impact overall survival and clinical improvement ${ }^{1,5)}$. In addition, there are a limited number of studies that address the role of surgery for spinal melanoma metastasis ${ }^{6}$. . There is no clear treatment for this reason, but there are studies as follows. One study reported that the number of melanoma metastatic sites is an important prognostic factor, and total tumor removal should be considered in healthy patients with spinal metastasis and surgically resectable tumors limited to one organ ${ }^{8)}$. Another study established that if the entire tumor cannot be removed, direct decompressive surgery plus postoperative RTx for all types of spinal metastasis is superior to radiotherapy alone ${ }^{4)}$. And surgical resection appears to preserve neurological function and can improve overall outcomes from melanoma metastatic to the spine ${ }^{6}$.

Spinal metastasis of choroid malignant melanoma has been

Table 1. Previous cases of spinal metastasis of choroid malignant melanoma

\begin{tabular}{|c|c|c|c|c|c|c|}
\hline $\begin{array}{l}\text { Sex/ } \\
\text { Age }\end{array}$ & Origin & $\begin{array}{l}\text { Duration from origin } \\
\text { to meta development }\end{array}$ & Lab & Operation & $\begin{array}{c}\text { Postoperation } \\
\text { treatment }\end{array}$ & Prognosis \\
\hline$M / 61$ & Left eye & 3 years & $\mathrm{LDH} 1,716 \mathrm{IU} / \mathrm{L}$ & $\begin{array}{l}\text { T10 laminectomy + tumor removal } \\
+ \text { pedicle fixation T8-9-10-11-12 }\end{array}$ & Expired & $\begin{array}{l}\text { Died a few weeks } \\
\text { later }\end{array}$ \\
\hline $\mathrm{F} / 54$ & Left eye & 20 years & & $\begin{array}{l}\text { T9 laminectomy + tumor removal } \\
+ \text { pedicle fixation T7-18-10-11 }\end{array}$ & $\begin{array}{l}\text { RTx } 15 \text { days, } \\
\text { CTx } 1 \text { cycle }\end{array}$ & $\begin{array}{l}\text { Multiple metastases } \\
\text { after } 7 \text { months }\end{array}$ \\
\hline
\end{tabular}

M: male; F: female; LDH: lactate dehydrogenase; T: thorax; RTx: radiotherapy; CTx: chemotherapy. 
reported in 3 cases, and specific treatment were presented in 2 cases. In 2 cases, tumor removal was performed, but the prognosis was poor (Table 1). One patient died several weeks after surgery, thus RTx and CTx were not performed. And another patient was performed RTx and CTx, but developed multiple metastasis after 7 months.

In our case, due to lack of follow-up, metastasis was not detected early and the patient had already multiple metastasis. Based on the results of previous studies, the tumors were not totally removed and decompressive tumor removal was performed. As in the previous 2 cases, L3 laminectomy, left facetectomy, and partial tumor removal with posterior pedicle screw fixation were performed. After surgery, RTx and CTx are being performed at other hospitals, and they have survived more than 1 year after surgery.

Metastasis caused by choroidal melanoma usually occurs within 5 years after diagnosis, but late metastasis is also a well-known complication. Shields et al. reported a case of orbital recurrence and liver metastasis 42 years after primary tumor enucleation ${ }^{7)}$. In ocular melanoma, the liver is the most common site of metastasis, and only one case of spine metastasis without liver metastasis has been reported ${ }^{3)}$.

In this case, if the metastasis had been identified earlier, it could have been limited to the liver and treated before metastasis to the spine.

\section{CONCLUSION}

We report a rare case of spinal metastasis of malignant choroid melanoma. To our knowledge, this is the first report of the lumbar spine as the main lesion location without thoracic or cervical spine symptoms. Unfortunately, due to a lack of follow-up, the metastasis was not detected and treated early. Because melanoma is associated with recurrence, we suggest that future cases of melanoma, even cases that achieve re- mission, complete follow-up over the patient's lifetime to detect and treat any recurrence.

\section{CONFLICTS OF INTEREST}

No potential conflict of interest relevant to this article was reported.

\section{REFERENCES}

1. Augsburger JJ, Corrêa ZM, Shaikh AH: Effectiveness of treatments for metastatic uveal melanoma. Am J Ophthalmol 148: 119-127, 2009

2. Hwang JK, Kim KH, Kim JS, Ahn SJ, Kuh SU: Late-developing metastatic malignant melanoma in the thoracic spine originating from choroidal melanoma. Korean J Spine 14:53-56, 2017

3. Mandaliya H, Singh N, George S, George M: Choroid melanoma metastasis to spine: A rare case report. Case Rep Ophthalmol Med 2016:2732105, 2016

4. Patchell RA, Tibbs PA, Regine WF, Payne R, Saris S, Kryscio RJ, et al.: Direct decompressive surgical resection in the treatment of spinal cord compression caused by metastatic cancer: a randomised trial. Lancet 366:643-648, 2005

5. Rantala ES, Hernberg M, Kivelä TT: Overall survival after treatment for metastatic uveal melanoma: A systematic review and meta-analysis. Melanoma Res 29:561-568, 2019

6. Shakur SF, Takagi I, Lukas RV, Chmura S, Gajewski TF, Roitberg BZ: Ocular melanoma metastasis to the cervical spine. J Clin Neurosci 19:610-611, 2012

7. Shields JA, Augsburger JJ, Donoso LA, Bernardino VB, Jr., Portenar M: Hepatic metastasis and orbital recurrence of uveal melanoma after 42 years. Am J Ophthalmol 100:666-668, 1985

8. Spiegel DA, Sampson JH, Richardson WJ, Friedman AH, Rossitch E, Hardaker WT, Jr., et al.: Metastatic melanoma to the spine. Demographics, risk factors, and prognosis in 114 patients. Spine (Phila Pa 1976) 20:2141-2146, 1995 